\title{
Flora bacteriana cloacal y nasal de Lepidochelys olivacea (Testudines: Cheloniidae) en el pacífico norte de Costa Rica
}

\author{
Mario Santoro ${ }^{1}$, Carlos Mario Orrego ${ }^{2}$ \& Giovanna Hernández Gómez ${ }^{3}$ \\ 1 Laboratorio de Parasitología, Escuela de Medicina Veterinaria, Universidad Nacional, Heredia, Costa Rica. marisant@libero.it \\ 2 Programa Regional Manejo de Vida Silvestre, Universidad Nacional, Heredia, Costa Rica. carlosmario@hotmail.com \\ 3 Laboratorio de Bacteriología, Escuela de Medicina Veterinaria, Universidad Nacional, Heredia, Costa Rica. \\ giovah@ns.medvet.una.ac.cr
}

Recibido 21-I-2003. Corregido 16-XII-2004. Aceptado 05-VII-2005.

\begin{abstract}
Cloacal and nasal bacterial flora of Lepidochelys olivacea (Testudines: Cheloniidae) from the North Pacific coast of Costa Rica. The aerobic cloacal and nasal bacterial flora of 45 apparently healthy female olive ridley sea turtles (Lepidochelys olivacea) was studied at Nancite nesting beach, in Santa Rosa National Park (Costa Rican North Pacific) during July and August 2002. Bacterial samples were obtained by inserting sterile swabs directly into the cloaca and the nasal cavities of the turtles. Ninety-nine aerobic bacterial isolates, including 10 Gram-negative and 5 Gram-positive bacteria, were recovered. The most common bacteria cultured were Aeromonas spp. (13/45) and Citrobacter freundi (6/45) from cloacal samples and Bacillus spp. (32/45), Staphylococcus aureus (6/45) and Corynebacterium spp. (5/45) from nasal ducts. The results of the present study showed that the aerobic bacterial flora of nesting female olive ridleys was composed of several potential human and animal microbe pathogens. Rev. Biol. Trop. 54(1): 43-48. Epub 2006 Mar 31.
\end{abstract}

Key words: Lepidochelys olivacea, olive ridley sea turtle, bacterial flora, bacterial disease, Nancite, Costa Rica.

Durante muchos años las especies de tortugas marinas han sufrido una fuerte disminución en sus poblaciones por varias causas, principalmente antrópicas: consumo de huevos y carne, uso del carapazón para la fabricación de artesanías, captura accidental en redes de pesca y palangres, colisiones con barcos, contaminación y destrucción del hábitat de alimentación y de desove (National Research Council 1990). Poco se conoce sobre el impacto de las enfermedades infecciosas bacterianas en las poblaciones de tortugas marinas en estado natural y sobre el papel que varios microorganismos puedan desarrollar como agentes patógenos (Glazebrook y Campbell 1990b). Muchas bacterias han sido identificadas como las causantes de enfermedades en tortugas marinas mantenidas en cautividad (Keymer 1978, Glazebrook et al. 1981, Lauckner 1985, Glazebrook y Campbell 1990a, Glazebrook et al. 1993). Sin embargo, se conoce que muchos microorganismos pueden ser causa de elevada mortalidad en otras especies de animales marinos de vida libre (Medway 1980, Ghittino et al. 1984, Gulland 1999). Además, muchas de estas bacterias son patógenas para los humanos. Recientemente, Vibrio mimicus en Costa Rica (Campos et al. 1996) y Salmonella chester en Australia (O`Grady y Krause 1999) fueron asociadas a enfermedades humanas por consumo de huevos y carne de tortugas marinas, respectivamente. Este trabajo tiene el objetivo de determinar la flora aerobia microbiana, nasal y cloacal, de la tortuga lora (Lepidochelys olivacea). 


\section{MATERIALES Y MÉTODOS}

Se recolectaron muestras bacteriológicas de 45 hembras (promedio largo curvo carapazón $68.16 \mathrm{~cm}$ ), entre los meses de julio y agosto del 2002 , en Playa Nancite $\left(10^{\circ} 48^{\prime} 17^{\prime \prime} \mathrm{N}\right.$, $85^{\circ} 41^{\prime} 58^{\prime \prime}$ W), Parque Nacional Santa Rosa, provincia de Guanacaste, Costa Rica.

En la noche, mientras se realizaba el desove se introdujeron hisopos estériles directamente en la cloaca, frotando entre la mucosa de la pared. Luego, después de haber limpiado la arena de la parte externa del rostro con un paño de papel estéril desechable, hisopos estériles se introdujeron en uno de los conductos nasales.

Todas las muestras fueron puestas en medio de transporte Stuart's (Difco) y llevadas, en menos de $24 \mathrm{~h}$ en un contenedor térmico con hielo al Laboratorio de Bacteriología de la Escuela de Medicina Veterinaria, Universidad Nacional, Heredia, Costa Rica. El material obtenido de los hisopos nasales se cultivó en agar sangre (Oxoid), agar MacConkey (Difco) y agar manitol sal (BBL).

Los hisopos cloacales se cultivaron en agar sangre, agar MacConkey y agar XLD (Oxoid). Además, para detectar la presencia de Salmonella, las muestras cloacales fueron incubadas a $37^{\circ} \mathrm{C}$ por $18 \mathrm{~h}$ en medio de enriquecimiento Rappaport-Vassiliadis-Soya peptone (RVS) Broth (Oxoid), luego sembradas en medio de cultivo XLD agar. Las placas se incubaron a $27^{\circ} \mathrm{C}$ aeróbicamente y después de $24 \mathrm{~h}$ fueron examinadas. Si el crecimiento bacteriano en este tiempo resultaba escaso o nulo, las placas se dejaron incubando por otras $24 \mathrm{~h}$.

Cada colonia fue separada y aislada en placas de agar sangre, clasificadas por la reacción de Gram y finalmente identificadas con pruebas bioquímicas rutinarias (Bisping y Amtsberg 1988). Algunos aislamientos fueron identificados hasta género y otros hasta especie. La identificación de las bacterias Gram-negativas se confirmó usando el sistema Api (bio-Merieux). Después del desove, todas las tortugas fueron sometidas a un examen clínico externo y solamente los resultados obtenidos de los análisis de las hembras consideradas aparentemente sanas fueron incluidas en este estudio.

\section{RESULTADOS}

De las muestras cultivadas se obtuvo un total de 99 aislamientos bacterianos, incluyendo 10 grupos de Gram-negativos y 5 de Gram-positivos. Del total, 35 bacterias fueron encontradas en la cloaca y 64 en las cavidades nasales. Nueve grupos bacterianos se aislaron en la cloaca y 11 grupos en las cavidades nasales, 5 grupos se encontraron en los dos sitios anatómicos de muestreo (Cuadro 1). Aeromonas spp. fue el microorganismo más frecuentemente aislado en la cloaca (13/45), seguido de Citrobacter freundi (6/45), Salmonella spp. (3/45), Acinetobacter spp. (3/45) y Pseudomonas aeruginosa (3/45).

Bacillus spp. fue el más frecuente hallado en las cavidades nasales (32/45), seguido de Staphylococcus aureus (6/45), Corynebacterium spp. (5/45), Pseudomonas spp. (4/45), Acinetobacter spp. (4/45), Proteus mirabilis, Aeromonas spp. y Lactobacillus spp. (3/45).

De cada tortuga se obtuvieron un promedio de 0.7 bacterias de la cloaca y 1.4 de las cavidades nasales. En las muestras cloacales predominaron los microorganismos Gramnegativos (33/35), mientras que en las primeras vías respiratorias los Gram-positivos (46/64). Enterococcus faecalis fue el único microorganismo Gram-positivo hallado en la cloaca.

\section{DISCUSIÓN}

El significado de la flora bacteriana que se puede encontrar en reptiles se entiende solo cuando se considera la interacción que existe entre los hospederos y el medio ambiente. Snipps et al. (1980) y Lawrence y Needham (1985) observaron como las especies de bacterias que se aíslan en tortugas en buen estado de salud frecuente no se diferencian significativamente de las que se encuentran en tortugas 
CUADRO 1

Clases de bacterias obtenidas a partir de hisopos de la cloaca (C) y conductos nasales (CN) de tortugas lora $(n=45)$

Bacterias

1) Acinetobacter spp.

2) Aeromonas spp.

3) Alcaligenes faecalis

4) Bacillus spp.

5) Citrobacter freundi

6) Corynebacterium spp.

7) Enterobacter agglomerans

8) Enterococcus faecalis

9) Escherichia coli

10) Lactobacillus spp.

11) Proteus mirabilis

12) Pseudomonas aeruginosa

13) Pseudomonas spp.

14) Salmonella spp.

15) Staphylococcus aureus

$\begin{array}{cc}\mathrm{C} \% & \mathrm{CN} \% \\ \text { (n. positivas) } & \text { (n. positivas) } \\ 6.6 \%(3) & 8.8 \%(4) \\ 28.8 \%(13) & 6.6 \%(3) \\ 2.2 \%(1) & 0 \\ 0 & 71.1 \%(32) \\ 13.3 \%(6) & 0 \\ 0 & 11.1 \%(5) \\ 0 & 4.4 \%(2) \\ 4.4 \%(2) & 0 \\ 6.6 \%(3) & 2.2 \%(1) \\ 0 & 6.6 \%(3) \\ 2.2 \%(1) & 6.6 \%(3) \\ 6.6 \%(3) & 2.2 \%(1) \\ 0 & 8.8 \%(4) \\ 6.6 \%(3) & 0 \\ 0 & 13.3 \%(6)\end{array}$

cautividad, han sido asociadas a enfermedad cutánea septicemica ulcerativa, dermatitis papilar, dermatitis focal erosiva, dermatitis ulcerosa traumática, dermatitis necrosante, estomatitis ulcerosa, rinitis obstructiva, queratoconjuntivitis, septicemia, bronconeumonía y osteomielitis (Lauckner 1985, Glazebrook y Campbell 1990a, Glazebrook et al.1993).

Un grupo de enfermedades debidas a la infección por Vibrio alginolyticus, Aeromonas hydrophila y Flavobacterium spp., conocidas como Estomatitis Ulcerativa-Rinitis Obstructiva-Bronconeumonía, fue la causa de altas tasas de mortalidad, próximas al $70 \%$, en ejemplares juveniles de Caretta caretta y Chelonia mydas mantenidas en cautividad (Glazebrook et al.1993). Corynebacterium spp. fue asociado a encefalitis de carácter multifocal en ejemplares juveniles de Caretta caretta (George et al. 1995).

Existen más de 2300 serotipos de Salmonella, muchos de los cuales fueron encontrados en reptiles silvestres y en cautiverio (Mermin et al. 1997), y algunos de estos 
en quelonios marinos (Keymer et al. 1968, Keymer 1978, Glazebrook y Campbell 1990a, Raidal et al. 1998, O’Grady y Krause 1999). Estos microorganismos son conocidos como causa de enfermedades oportunistas principalmente cuando los hospederos están debilitados y ocasionalmente como causa de gastroenteritis en los seres humanos por consumo de carne de tortuga (O’Grady y Krause 1999). Se considera actualmente que las tortugas marinas no son vectores de Salmonella como ocurre con otros reptiles (Johnson-Delaney 1996).

En reptiles terrestres, coliformes y Enterobacter spp. son asociados a enfermedades locales y generales (Hoff et al. 1984, Glazebrook y Campbell 1990a). Proteus mirabilis y Bacillus spp. son considerados saprófitas $\mathrm{y}$ parte de la flora normal que se encuentra sobre la piel de tortugas marinas (Glazebrook y Campbell 1990a).

Bacterias del género Vibrio frecuentemente aisladas a partir de diferentes tipos de muestras de tortugas marinas y asociadas a varias patologías en este grupo de reptiles (Glazebrook et al.1981, 1993, Aguirre et al. 1984, Obendorf et al. 1987, Campos et al. 1996, Acuña et al. 1999), no fueron halladas en este estudio, utilizando métodos de cultivo y de aislamiento convencionales (Quinn et al. 1999, Carnahan y Andrews 2000).

Los estudios de la flora bacteriana en animales de vida libre son de fundamental importancia pues permiten conocer el papel que juegan estos microorganismos en las enfermedades infecciosas y como afectan la supervivencia de las poblaciones de tortugas marinas en condiciones naturales dentro del equilibrio biológico de estos vertebrados. Considerando que el número de los microorganismos resulta mayor en reptiles con inestable estado de salud, pensamos que este aspecto puede ser utilizado como un importante índice para identificar grupos de animales inmunodeprimidos o enfermos, evaluando la flora bacteriana de una población durante varios años. En fin, el conocimiento de la flora indígena de animales silvestres es importante para entender los riesgos potenciales de la zoonosis.
En esta investigación demostramos, en hembras de tortugas lora aparentemente sanas, la presencia de un amplio espectro de bacterias, muchas de las cuales son patógenas para el ser humano y animales.

\section{AGRADECIMIENTOS}

Nuestro agradecimiento al Área de Conservación Guanacaste por su apoyo en el uso de las infraestructuras. A Humberto Cedeño, Ana Jiménez, Magaly Caballero, Javier González de la Escuela de Medicina Veterinaria, Heredia, Costa Rica, por su apoyo y valiosa colaboración durante la realización de esta investigación.

\section{RESUMEN}

Con el objetivo de determinar la flora normal aerobia, cloacal y nasal de la tortuga lora (Lepidochelys olivacea), entre los meses de julio y agosto del 2002, se colectaron muestras bacteriológicas de 45 quelonios aparentemente sanos, durante el desove en Playa Nancite, Parque Nacional Santa Rosa, Costa Rica, a través del uso de hisopos estériles que se introdujeron en la cloaca y en uno de los conductos nasales. De las muestras recolectadas se obtuvieron e identificaron un total de 99 aislamientos, incluyendo 10 grupos de Gram-negativos y 5 de Gram-positivos. De cada tortuga se obtuvo un promedio de 0.7 bacterias de la cloaca y 1.4 de las cavidades nasales. Las bacterias más frecuente halladas fueron Aeromonas spp. (13/45) y Citrobacter freundi (6/45) en la cloaca, y Bacillus spp. (32/45), Staphylococcus aureus (6/45) y Corynebacterium spp. (5/45) en las cavidades nasales. En este investigación, la flora microbiana de las tortugas lora resultó constituida por microorganismos potencialmente patógenos para el ser humano y las tortugas.

Palabras clave: Lepidochelys olivacea, tortuga lora, flora bacteriana, enfermedades bacterianas, Nancite, Costa Rica.

\section{REFERENCIAS}

Acuña, M.T., G. Díaz, H. Bolaños, C. Barquero, O. Sánchez, L.M. Sánchez, G. Mora, A. Chávez \& E. Campos. 1999. Source of Vibrio mimicus contamination of turtle eggs. Appl. Environ. Microbiol. 65: 336-338. 
Aguirre, A.A., G.H. Balazs, B. Zimmerman \& T.R. Spraker. 1994. Evaluation of Hawaii green turtles (Chelonia mydas) for potential pathogens associated with fibropapillomas. J. Wildl. Dis. 30: 8-15.

Bisping, W. \& G. Amtsberg. 1988. Colour Atlas for the diagnosis of bacterial pathogens in animals. Paul Parey Scientific Publishers (ed.). Berlin and Hamburg. 339 p.

Campos, E., H. Bolaños, M.T. Acuña, G. Díaz, M.C. Matamoros, H. Raventós, L.M. Sánchez, O. Sánchez, C. Barquero \& Red Nacional de Laboratorios para Cólera, Costa Rica. 1996. Vibrio mimicus diarrhea following ingestion of raw turtle eggs. Appl. Environ. Microbiol. 62: 1141-1144.

Carnahan A.M. \& G. Andrews. 2000. Vibrio, Aeromonas, Plesiomonas and Campylobacter species, p. 515537. In Mahon C.R. \& G. Manuselis (ed.). Textbook of diagnostic microbiology. W.B. Saunders Co., Philadelphia, Pennsylvania.

Dickinson, V.M., T. Duck, C.R. Schwalbe, J.L. Jarchow \& M.H. Trueblood. 2001. Nasal and cloacal bacteria in free-ranging desert tortoises from the western United States. J. Wildl. Dis. 37: 252-257.

George, R.H., R.E. Wolke \& J.A. Keinath. 1995. A new reason why apparently healthy sea turtles strand in warm weather. Proc. 12th Annu. Workshop on Sea Turtle Conservation and Biology. In J.I. Richardson \& T.H. Richardson (compilers). N.M.F.S. Tech. Memo., NOAA-TM-NMFS-SEFSC-361, Miami, Fl. 41 p.

Ghittino, F., H. Schwedler \& P. De Kinkelin. 1984. The principal infectious diseases of fish and their general control measures. In P. De Kinkelin (ed.). Symposium on fish vaccination. Publicacion Office Internationale des Epizootics, Paris, France, p. 5-38.

Glazebrook, J.S., R.S.F. Campbell. \& R.H. Johnson. 1981. Traumatic ulcerative dermatitis: A disease of captive sea turtle Chelonia mydas (L) in north-east Australia. In M.E. Fowler (ed.). Wildlife diseases of the Pacific basin and other countries. Fruitridge Printing, Sacramento, California, p.160.

Glazebrook, J.S. \& R.S.F. Campbell. 1990a. A survey of the diseases of marine turtles in northern Australia. I. Farmed turtles. Dis. Aquat. Org. 9: 83-95.

Glazebrook, J.S. \& R.S.F. Campbell. 1990b. A survey of the diseases of marine turtles in northern Australia. II. Oceanarium-reared and wild turtles. Dis. Aquat. Org. 9: 97-104.

Glazebrook, J.S., R.S.F. Campbell \& A.T. Thomas. 1993. Studies on an ulcerative stomatitis - obstructive rhinitis - pneumonia disease complex in hatchling and juvenile sea turtles, Chelonia mydas and Caretta caretta. Dis. Aquat. Org. 16: 133-147.

Gulland, F.M.D. 1999. Leptospirosis in Marine Mammals, p. 469-471. In M.E. Fowler and R.E. Miller (ed.). Zoo and Wild Animal Medicine, Current Therapy 4, Saunders, Philadelphia, Pennsylvania.

Hoff, G.L., F.L. Frye \& E.R. Jacobson. 1984. Diseases of amphibians and reptiles. Plenum, New York. $784 \mathrm{p}$.

Jacobson, E.R., J.L. Behler \& J.L. Jarchow. 1998. Health assessment of chelonians and release into the wild, p. 232-242. In M.E. Fowler \& R.E. Miller (ed.). Zoo and Wild Animal Medicine, Current Therapy 4, W.B. Saunders Co., Philadelphia, Pennsylvania.

Jacobson, E.R., J.M. Gaskin, M.B. Brown, R.K. Harris, C.H. Gardiner, J.L. La Pointe, H.P. Adams \& C. Reggiardo. 1991. Chronic upper respiratory tract disease of free-ranging desert tortoises (Xerobates agassizii). J. Wildl. Dis. 27: 296-316.

Johnson-Delaney, C.A. 1996. Reptile Zoonoses and threats to public health, p. 20-33. In D.R. Mader (ed.). Reptile Medicine and Surgery, Saunders, Philadelphia, Pennsylvania.

Keymer, I.F., D. Ridealgh \& G. Fretwell. 1968. Salmonella regent: a new species associated with colitis in a Pacific hawksbill turtle (E. Imbricata bissa). J. Path. Bact. 96: 215.

Keymer, I.F. 1978. Diseases of chelonians: (2) Necropsy survey of terrapin and turtles. Vet. Rec. 103: 577-582.

Lauckner, G. 1985. Diseases of Reptilia, p. 561-566. In O. Kinne (ed.). Diseases of marine animals, Vol. IV, part. 2. Biologische Anstalt Helgoland, Hamburg, Germany.

Lawrence, K. \& J.R. Needdham. 1985. Rhinitis in long term captive Mediterranean tortoises (Testudo graecae and Testudo hermanni). Vet. Rec. 21: 662-664.

Medway, W. 1980. Some bacterial and mycotic diseases of marine mammals. J. Am. Vet. Med. Assoc. 177: 831-834.

Mermin, J., B. Hoar \& F.J. Angulo. 1997. Iguanas and Salmonella marina infection in children: a reflection of the increasing incidence of reptile-associated salmonellosis in the United States. Pediatrics. 99: 399-402.

National Research Council. 1990. Decline of the sea turtles: causes and prevention, National Academy Press, Washington, D.C. 280 p.

Obendorf, D.L., J. Carson \& T.J. Mcmanus. 1987. Vibrio damsela infection in a Stranded Leatherback Turtle (Dermochelys coriacea). J. Wildl. Dis. 23: 666-668. 
O’Grady, K.A. \& V. Krause. 1999. An outbreak of salmonellosis linked to a marine turtle. Southeast Asian J. Trop. Med. Public. Health. 30: 324-327.

Quinn, P.J., M.E. Carter, B. Markey \& G.R. Carter. 1999. Aeromonas, Plesiomonas and Vibrio Species, p. 243-258. In Mosby (ed.). Clinical Veterinary Microbiology, London, England.
Raidal, S.R., M. Ohara, R.P. Hobbs \& R.I. Prince. 1998. Gram-negative bacterial infections and cardiovascular parasitism in green sea turtles (Chelonia mydas). Aust. Vet. J. 76: 415-417.

Snipes, K.P., E.L. Biberstein \& M.E. Fowler. 1980. A Pasteurella spp. Associated with respiratory disease in captive desert tortoises. J. Am. Vet. Med. Assoc. 177: 804-807. 